\title{
Rate and correlates of weight change in Huntington's disease
}

\author{
J M Hamilton, T Wolfson, G M Peavy, M W Jacobson, J Corey-Bloom
}

J Neurol Neurosurg Psychiatry 2004;75:209-212. doi: 10.1136/jnnp.2003.017822

See end of article for authors' affiliations .....................

Correspondence to: Dr Joanne M Hamilton, Department of

Neurosciences, 9500

Gilman Drive (0948), La

Jolla, CA 92093-0948,

USA (for the investigators of the Huntington Study

Group*); jmhamilton@ ucsd.edu

Received 29 April 2003

In revised form

18 June 2003

Accepted 21 June 2003

\begin{abstract}
Objective: To determine the rate and correlates of weight change in a large, well characterised sample of patients with Huntington's disease followed at 44 sites by the Huntington Study Group.

Participants and methods: Weight change was assessed in 927 adults with a definite diagnosis of Huntington's disease who were followed prospectively for (mean (SD)) 3.4 (1.4) years. The unified Huntington's disease rating scale was used to assess weight, motor dysfunction (including chorea and dystonia), depressive symptoms, and functional decline.

Results: Random effects modelling determined that patients gained an average of 0.11 (1.7) kg/year and their chorea scores increased by $0.36(0.78)$ points/year. There were significant but weak relations between weight loss and increasingly severe chorea $(r=-0.13)$, worse baseline motor performance $(r=-0.12)$, less severe baseline depressed mood $(r=0.14)$, and poorer baseline independence ratings $(r=0.07)$. Patients who were within 0 to 2 years of symptom onset at the time of the baseline visit gained more weight than those with longer disease duration.

Conclusions: Weight loss following symptom onset is not a consistent feature of Huntington's disease. The mechanisms contributing to weight change in this condition are unclear and probably multifactorial. Future studies examining asymptomatic carriers of the mutation could be helpful in identifying incipience of low body weight and may be better suited for identifying clinical correlates of weight loss than studies in symptomatic patients.
\end{abstract}

$\mathrm{H}$ untington's disease results in a severe movement disorder (that is, chorea, dystonia, bradykinesia) accompanied by notable cognitive impairment (for example, executive dysfunction) and behavioural changes such as depression, irritability, apathy, and inflexibility. ${ }^{1}$ In addition, the disease has been associated with significant weight loss, ${ }^{23}$ even though affected individuals often report increased appetite and appear to consume more energy than non-affected controls. ${ }^{34}$ A survey of 1283 caregivers of patients with Huntington's disease indicated that weight loss became common in the middle to late stages of the disease. ${ }^{5}$ Despite this report, ${ }^{5}$ affected patients have been found to be significantly underweight at the time of their first neurological examination, ${ }^{67}$ suggesting that weight loss (or failure to gain weight) may occur earlier in the course of the disease than the caregivers acknowledged. It is unlikely that choreiform movements explain this early feature of the disease, because recently diagnosed patients have lower body mass indices than sex and age matched control subjects ${ }^{7}$ even though they have minimal chorea. However, in more advanced stages of the disease, weight loss may be most severe in ambulatory patients with unremitting chorea, ${ }^{8}$ presumably because of the additional sedentary energy expenditure caused by involuntary movements. ${ }^{9}$

While Djoussé and colleagues ${ }^{7}$ have recently shown that low body weight is common in early Huntington's disease, the rate of weight change has not been systematically examined in a large clinical sample to determine whether this is a significant feature of Huntington's disease and at what point in the course of the disease it is most likely to occur. The present study examined longitudinal weight data from a large cohort of patients with Huntington's disease followed by the Huntington Study Group (HSG) to identify

*See appendix (web only) for a complete list of the members of the Huntington Study Group. the rate and correlates of weight change during the course of the disease. Given previous reports, ${ }^{23}$ we hypothesised that affected individuals would lose weight over the course of the study and that increasingly severe chorea (measured across several visits) would be associated with greater weight loss.

\section{METHODS}

\section{Subjects}

Participants included 927 adults (472 women and 455 men) who had a definite diagnosis of Huntington's disease confirmed by characteristic signs of the disease accompanied by a positive family history or the presence of expanded CAG repeats in IT15 on chromosome 4p, or both. The patients completed their baseline evaluations between 20 September 1993 and 25 August 1999, at one of 44 HSG sites across the USA, Canada, Europe, and Australia. From the HSG database, subjects with the following characteristics were selected:

- manifest symptoms of Huntington's disease at the baseline visit;

- aged 18 years old or older;

- valid weight measurement at baseline;

- completion of at least two additional visits at roughly six monthly intervals.

Patients represented a broad range of disease duration and severity. They were followed for a mean (SD) period of 3.4 (1.4) years, with visits separated by an average of 8.4 (6.2) months, and all participants had at least three follow up examinations (mean 5.8 (2.8)). Informed consent was obtained through the institutional review boards at participating sites.

Abbreviations: BMI, body mass index; HSG, Huntington Study Group; TFC, total functional capacity; UHDRS, unified Huntington's disease rating scale 


\section{Measures}

The patients' signs and symptoms were rated using the unified Huntington's disease rating scale (UHDRS) ${ }^{10}$ as part of their ongoing clinical care and evaluation. HSG sites mailed the UHDRS forms to the Coordination Center at the University of Rochester. Individuals were identified by numerical code only. Demographic data included date of birth, height in meters $(\mathrm{m})$, and sex. The UHDRS medical history form provided additional information regarding symptom or sign onset. First symptoms or signs included motor, oculomotor, cognitive, or psychiatric changes. Duration of illness was calculated as the difference between the date of initial evaluation and date of symptom or sign onset, as judged by the evaluator. Weight in kilograms was measured at each visit, and the body mass index (BMI; kg/ $\mathrm{m}^{2}$ ) was calculated for individuals whose height had been recorded during an evaluation $(\mathrm{n}=801)$.

The components of the UHDRS that assess motor performance, the severity and frequency of depressed mood, and functional capacity were included in the analyses.

The motor examination includes an evaluation of oculomotor dysfunction, dysarthria, tongue impersistence, dysdiadochokinesia, motor sequencing, dystonia, bradykinesia, chorea, gait disturbance, and postural instability. The severity of motor abnormalities is rated on a five point scale $(0=$ absent, $4=$ severe $)$; thus total scores range between 0 and 124, with higher numbers signifying greater motor dysfunction. For the purposes of this study, motor performance was examined both overall and specifically for severity of chorea or dystonia. The chorea subscale rated the severity of choreiform movements in the face, mouth, trunk, arms, and legs, with a maximum severity of 28 points. The dystonia subscale rated the severity of dystonia in the trunk, arms, and legs with a maximum severity of 20 points.

The mood assessment was derived from the 1994 version of the UHDRS. It rates the severity and frequency of depressed mood (that is, feeling sad, sad voice/expression, tearfulness, inability to enjoy anything) as reported by the patient and/or a caregiver on a five point scale $(0=$ absent/almost never, $4=$ severe/almost always). The severity and frequency ratings were multiplied to yield a single score ranging from 0 to 16.

Functional ratings included the total functional capacity (TFC) scale and the independence scale. The TFC scale includes five items (occupation, finances, domestic chores, $\mathrm{ADL}$, and care level) that are rated on a two or three point scale. Possible scores range from 0 (severe impairment) to 13 (no impairment). The independence scale rates independence in performing activities of daily living on a scale from 0 to 100, with higher scores indicating greater independence.
Table 1 details the scores for all measures at the initial baseline visit.

\section{Statistics \\ Rate of change}

The annual rate of change of weight and chorea severity were estimated using a random effects model ${ }^{11}{ }^{12}$ for the entire sample and for men and women separately. That is, for each of the subjects we estimated an intercept and a slope across time for the response variables (weight and chorea). The individual slopes served as our measures of change and were averaged to reveal the rate of change (per year) for the sample. The repeated measures software was programmed by Dr I Abramson of the mathematics department at the University of California, San Diego. ${ }^{12}$ It is a flexible collection of routines in the S-Plus ${ }^{13}$ language, which is insensitive to the problems of imbalance, erratic time schedules, and missing values that frustrate or defeat standard software. We can provide a thorough mathematical description of the procedure on request.

To determine the pattern of weight change associated with duration of illness at the initial assessment visit, duration of illness was divided into five categories ( 0 to 2 years, 2 to 5 years, 5 to 10 years, 10 to 15 years, and $15+$ years). Rate of weight change was then submitted to an analysis of variance (ANOVA) with Tukey's LSD post-hoc analyses to determine whether the pattern of weight change varied reliably with duration of illness at the baseline visit. Independent samples $t$ tests were used to examine more closely the differences in clinical characteristics of patients who gained at least $1 \mathrm{~kg} /$ year and those who lost at least $1 \mathrm{~kg} /$ year.

\section{Correlates of weight change}

The relation between the annual rate of weight change and the annual rate of change in chorea severity was investigated using Pearson product-moment correlation analysis. Pearson product-moment correlations also tested the strength of association between the annual rate of weight change and baseline age, weight, total motor score, chorea, dystonia, depressed mood, and daily functioning on the TFC scale and the independence scale.

\section{RESULTS \\ Rate of weight change}

Results are given as mean (SD). Random effects modelling showed that the Huntington's disease sample as a whole gained an average of 0.11 (1.7) $\mathrm{kg} /$ year across the roughly three and a half year assessment period (fig l, left panel). The pattern of weight change was essentially identical when the

\begin{tabular}{|c|c|c|c|}
\hline Feature & $\begin{array}{l}\text { Huntington's disease } \\
\text { sample }\end{array}$ & Loss $\geqslant 1 \mathrm{~kg} /$ year & Gain $\geqslant 1 \mathrm{~kg} /$ year \\
\hline Age (years) & $48.0(12.0)$ & $48.6(12.3)$ & $48.6(11.0)$ \\
\hline Weight $(\mathrm{kg})$ & $68.8(14.7)$ & 70.6 (14.4) & $69.3(15.7)$ \\
\hline Height $(m)$ & $1.7(0.1)$ & $1.7(0.1)$ & $1.7(0.1)$ \\
\hline Body mass index $\left(\mathrm{kg} / \mathrm{m}^{2}\right)$ & $23.8(4.4)$ & $24.8(4.2)$ & $24.2(5.4)$ \\
\hline Duration of illness (years) & $7.3(5.0)$ & $7.7(5.2)$ & $7.1(5.0)$ \\
\hline Total motor score & $39.1(18.7)$ & $43.5(19.0)$ & $37.1(17.8)$ \\
\hline Chorea & $10.8(5.1)$ & $11.5(5.3)$ & $10.4(5.3)$ \\
\hline Dystonia & $3.3(4.0)$ & $3.6(4.0)$ & $3.1(3.9)$ \\
\hline Depressed mood rating & $3.0(3.6)$ & $2.8(3.5)$ & $4.1(3.9)$ \\
\hline Total functional capacity & $8.2(3.5)$ & $7.7(3.5)$ & $8.1(3.3)$ \\
\hline Independence scale & $79.9(16.8)$ & $76.3(18.6)$ & $80.7(15.8)$ \\
\hline Weight change (kg/year) & $0.1(1.7)$ & $-2.0(1.0)$ & $2.3(1.3)$ \\
\hline Chorea change (points/year) & $0.4(0.8)$ & $0.4(0.8)$ & $0.2(0.8)$ \\
\hline
\end{tabular}

Values are mean (SD). 

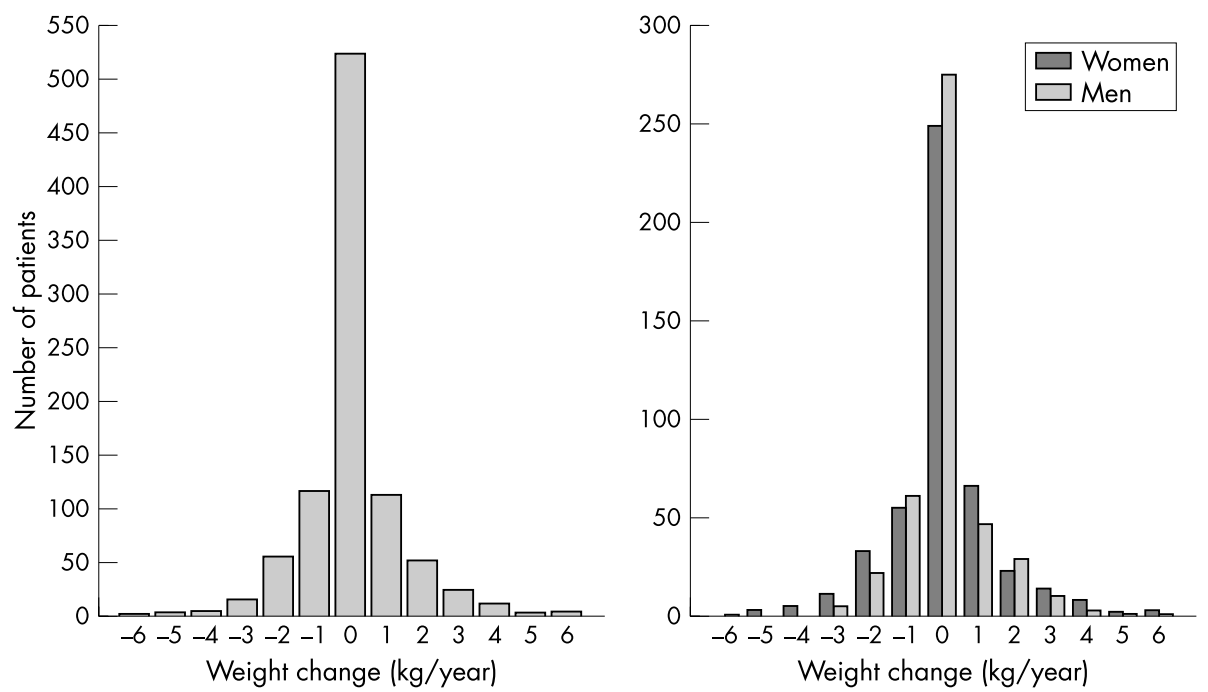

Figure 1 Frequency distribution of the annual rate of weight change ( $\mathrm{kg} /$ year) in the entire Huntington's disease sample (left) and stratified by sex (right). The bars represent the number of patients in each weight change category. It is apparent from this distribution that the majority of patients maintained a steady weight across the three and a half year study timeframe.

models were calculated separately by sex. Women gained an average of $0.11(1.8) \mathrm{kg} / \mathrm{year}$ and men an average of 0.10 (1.5) $\mathrm{kg} /$ year (fig l, right panel). There was a significant difference in the profile of weight change depending on the estimated duration of illness at the baseline visit $\left(\mathrm{F}_{(4,838)}=2.42 ; \mathrm{p}<0.05\right)$. Specifically, patients with the shortest estimated duration of illness ( 0 to 2 years) gained significantly more weight over the course of a year than all other groups except those who had had symptoms of Huntington's disease for 10 to 15 years (fig 2). There was no significant difference between these two groups.

\section{Clinical characteristics of patients who lost $v$ gained at least $1 \mathrm{~kg} /$ year}

In order to examine differences in the clinical characteristics of patients who specifically lost weight compared with those who gained weight, independent samples $t$ tests were used. As apparent from table 1 , there were no significant differences in age, estimated age of onset, duration of illness, baseline weight, or baseline scores on the TFC scale. The patients who lost $1 \mathrm{~kg}$ or more per year scored lower on the independence scale $\left(t_{(397)}=2.6, \mathrm{p}=0.01\right)$, had greater total motor dysfunction $\left(t_{(332)}=-3.2, \mathrm{p}<0.01\right)$, more baseline chorea $\left(t_{(397)}=-2.1, \mathrm{p}<0.05\right)$, a greater increase in chorea severity $\left(t_{(401)}=-2.5, \mathrm{p}=0.01\right)$, and a less depressed

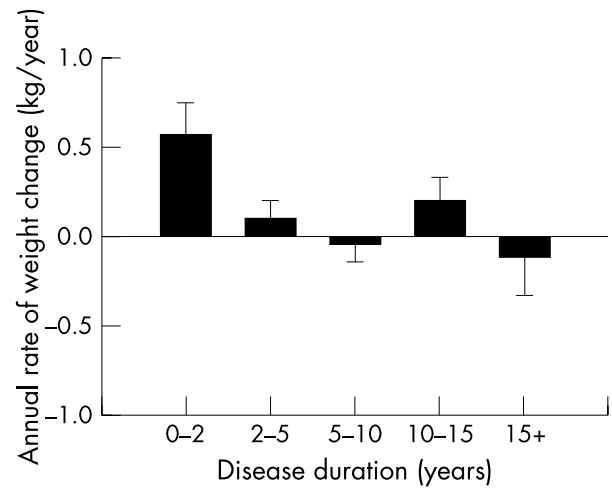

Figure 2 The mean annual rate of change in weight $(\mathrm{kg} /$ year) with respect to the length of time over which patients were affected by Huntington's disease before their baseline visit (disease duration). Standard error bars are included. $\operatorname{mood}\left(t_{(291)}=2.9, \mathrm{p}<0.01\right)$ than patients who gained at least $1 \mathrm{~kg} /$ year. It is notable, however, that the actual mean differences between these two groups were quite small, and thus would not be particularly useful for predicting which patients would develop problematic weight loss.

\section{Correlates of weight change}

To examine the question of whether increasingly severe chorea is related to greater weight loss, Pearson productmoment correlation analyses showed a statistically significant but weak relation between weight loss and chorea, such that greater weight loss was associated with worsening chorea $(r=-0.13 ; \mathrm{p}<0.001)$. With respect to the baseline measures, more severe motor impairment $(r=-0.12$; $\mathrm{p}=0.001)$, less severe depressed mood $(r=0.14$; $\mathrm{p}=0.001)$, and poorer independence ratings on the independence scale $(r=0.07 ; \mathrm{p}<0.05)$ were weakly related to greater weight loss.

\section{DISCUSSION}

Contrary to our hypothesis and to reports in smaller samples of patients with Huntington's disease, ${ }^{2}{ }^{3}$ the average patient in this large clinical sample netted a slight increase in weight over the study period, with the pattern of weight change being essentially identical for men and women. Importantly, while the patients in this sample gained on average $0.1 \mathrm{~kg} /$ year, this is less than the US national average of between 0.2 and $0.9 \mathrm{~kg} /$ year. $^{14-18}$ Furthermore, in comparison with data acquired in the US national health and nutrition examination survey (NHANES III), ${ }^{19}$ the patients in this sample weighed almost $10 \mathrm{~kg}$ less than a community sample of comparably aged adults. These results are consistent with those presented by Djoussé and colleagues, ${ }^{7}$ who showed that even in the earliest stages of Huntington's disease, patients weigh significantly less than healthy age matched controls.

A more detailed characterisation of weight change across disease duration found that patients who had developed signs of Huntington's disease most recently ( 0 to 2 years) gained more weight than patients who had been affected by the disease for longer. In fact, those patients who were very recently diagnosed with the disease showed a pattern of weight gain that was comparable with community samples. ${ }^{14-18}$ Minimal weight loss was evident in patients who had lived with Huntington's disease for 5 to 10 years before their baseline visit, consistent with findings from a caregiver's 
survey $^{5}$ reporting that weight loss begins in the later stages of the disease. It is notable that patients who had had Huntington's disease for 10 to 15 years at the point of their baseline visit showed a slight increase in weight. Based on clinical experience, this pattern may result from implementation of interventions directed at improving the patient's weight (for example, nutritional supplementation, admission to an institution, use of feeding tubes). Because these patients were followed at HSG sites, it is likely that their care providers were especially attuned to the importance of maintaining adequate body weight. It is possible that without such intervention the patients' weight change would have followed a clear downward slope (fig 2).

It has been suggested that patients who lose a substantial amount of weight do so because of an increase in involuntary energy expenditure secondary to chorea, ${ }^{9}$ and there are transgenic mice models that support this view. ${ }^{20}$ In our study, there was a statistically significant relation between increasing chorea severity and decreasing weight, but the strength of this relation did not provide compelling evidence to support the hypothesis that patients lose weight as their choreiform movements become more pronounced. A stronger relation may have been revealed if patients had been followed for longer than the three and a half year average, because in this time frame the annual rate of change on the chorea rating was minimal. However, there was a wide range in the baseline chorea ratings but still no relation between severity of chorea and weight change. The lack of a more robust relation indicates that the mechanisms contributing to weight change in Huntington's disease are likely to be multifactorial and probably result from interactions between voluntary and involuntary energy expenditure, the ability to maintain adequate energy intake, and potential abnormalities of intrinsic metabolic functioning.

Unfortunately, neither direct group comparisons nor correlational analyses revealed clinically meaningful correlates of weight loss. While weight loss was weakly associated with more motor dysfunction, less severely depressed mood, and greater functional dependence at baseline, the findings would be difficult to use to flag individuals at risk of problematic weight loss. The negligible clinical differences between those patients who actually lost $1 \mathrm{~kg}$ or more per year compared with those who gained $\mathrm{l} \mathrm{kg}$ or more emphasise this difficulty. It is quite possible that clinical information not available for this study-for example, the introduction of nutritional supplements, institutional placement, and types of drugs used-would be useful for explaining the pattern of weight change that we found here. For example, patients with greater motor dysfunction and functional dependence may lose more weight than less impaired patients, because dysphagia or difficulty in planning and initiating meals may make it harder for them to nourish themselves. The introduction of nutritional supplements or institutional placement could alter this situation.

In addition, drug use by these patients was not systematically recorded, but it is likely that many were taking agents that are known to cause weight gain (for example, neuroleptics or antidepressants). This limitation may be particularly important for interpreting the relation between depressed mood and weight change. Patients who are rated as being more depressed at baseline may have been treated with antidepressant drugs, and thus their weight gain may reflect the side effects of these drugs.

The relation between mood state and weight change deserves further evaluation with a more sensitive mood rating than the one that was available for this study. Our rating has not been validated as a quantitative measure, but rather serves descriptive purposes. A measure that includes a specific assessment of apathy as well as depression may provide meaningful information about individuals at risk of significant weight loss.

These ideas are obviously speculative, but deserve further study because the mechanisms related to problematic weight loss in Huntington's disease have not been identified. As a previous report has established that patients with Huntington's disease are much leaner than the normal population, even in the earliest stages of the disease, ${ }^{7}$ future studies examining asymptomatic carriers of the Huntington's disease mutation might pinpoint the development of low body weight and may be better suited than studies of symptomatic patients for identifying clinical correlates of malnutrition and weight loss.

\section{ACKNOWLEDGEMENTS}

Supported by NIH grant P50 AG05131, the Huntington Study Group, and a Huntington Disease Society of America center of excellence grant to the University of California at San Diego (UCSD).

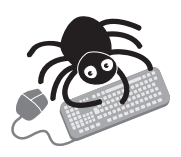

An appendix is provided on the JNNP website (www.jnnp.com/supplemental) giving a full list of the members of the Huntington Study Group.

\section{Authors' affiliations}

J M Hamilton, G M Peavy, J Corey-Bloom, Department of Neurosciences, University of California at San Diego, San Diego, California, USA

T Wolfson, Department of Family and Preventive Medicine, University of California at San Diego

M W Jacobson, Department of Psychiatry, University of California at San Diego

Competing interests: none declared

\section{REFERENCES}

1 Caine ED, Shoulson I. Psychiatric syndromes in Huntington's disease. Am J Psychiatry 1983;140:728-33.

2 Sanberg PR, Fibiger HC, Mark RF. Body weight and dietary factors in Huntington's disease patients compared with matched controls. Med J Aust 1981;1:407-9.

3 Farrer LA, Meaney FJ. An anthropometric assessment of Huntington's disease patients and families. Am J Phys Anthropol 1985;67:185-94.

4 Morales LM, Estevez J, Suarez H, et al. Nutritional evaluation of Huntington's disease patients. Am J Clin Nutr 1989:50:145-50.

5 Kirkwood SC, Su JL, Conneally M, et al. Progression of symptoms in the early and middle stages of Huntington's disease. Arch Neurol 2001;58:273-8.

6 Myers RH, Sax D, Koroshetz WJ, et al. Factors associated with slow progression in Huntington's disease. Arch Neurol 1991 48:800-4.

7 Dioussé L, Knowlton B, Cupples LA, et al. Weight loss in early stage of Huntington's disease. Neurology 2002;59:1325-30.

8 Stoy N, McKay E. Weight loss in Huntington's disease. Ann Neurol 2000;48:130-1.

9 Pratley RE, Salbe AD, Ravussin E, et al. Higher sedentary energy expenditure in patients with Huntington's disease. Ann Neurol 2000;47:64-70.

10 Huntington Study Group. Unified Huntington's disease rating scale: reliability and consistency. Mov Disord 1996;1 1:136-42.

11 Laird NM, Ware JH. Random-effects models for longitudinal data. Biometrics 1982;38:963-74.

12 Abramson I. A recursive regression for high dimensional models with application to growth curves and repeated measures. J Am Stat Assoc 1988:83:1073-7

13 Mathsoft Inc. S-PLUS. (5.1). 1999. Lucent Technologies Inc.

14 Williamson DF, Kahn HS, Remington PL, Anda RF. The 10-year incidence of overweight and major weight gain in US adults. Arch Intern Med 1990;150:665-72.

15 Shah M, Hannan PJ, Jeffery RW. Secular trend in body mass index in the adult population of three communities from the upper mid-western part of the USA: the Minnesota Heart Health Program. Int J Obes 1991;15:499-503.

16 Williamson DF. Descriptive epidemiology of body weight and weight change in US adults. Ann Intern Med 1993;1 19:646-9.

17 Jeffery RW, French SA. Preventing weight gain in adults: design, methods and one year results from the Pound of Prevention study. Int $J$ Obes 1997;21:457-64.

18 Lewis CE, Jacobs DR, McCreath $\mathrm{H}$, et al. Weight gain continues in the 1990s: 10 -year trends in weight and overweight from the CARDIA study. Coronary Artery Risk Development in Young Adults. Am J Epidemiol 2000;151:1172-81.

19 NHANES III. National Center for Health Statistics. Health, United States. 1995. Hyattsville: Public Health Service, 1996.

20 Mangiarini L, Sathasivam K, Seller M, et al. Exon 1 of the HD gene with an expanded CAG repeat is sufficient to cause a progressive neurological phenotype in transgenic mice. Cell 1996;87:493-506. 